\title{
Autocalibrating and Calibrationless Parallel Magnetic Resonance Imaging as a Bilinear Inverse Problem
}

\author{
(Invited Paper) \\ Martin Uecker \\ Diagnostic and Interventional Radiology \\ University Medical Center Göttingen, \\ Göttingen, Germany \\ and \\ German Center for Cardiovascular Research (DZHK) \\ partner site Göttingen, Germany \\ Email: martin.uecker@med.uni-goettingen.de
}

\begin{abstract}
Modern reconstruction methods for magnetic resonance imaging (MRI) exploit the spatially varying sensitivity profiles of receive-coil arrays as additional source of information. This allows to reduce the number of time-consuming Fourierencoding steps by undersampling. The receive sensitivities are a priori unknown and influenced by geometry and electric properties of the (moving) subject. For optimal results, they need to be estimated jointly with the image from the same undersampled measurement data. Formulated as an inverse problem, this leads to a bilinear reconstruction problem related to multi-channel blind deconvolution. In this work, we will discuss some recently developed approaches for the solution of this problem.

Index Terms-Magnetic resonance imaging, Compressed sensing, Nonuniform sampling, Image reconstruction, Calibration
\end{abstract}

\section{PARAllel Imaging}

The multi-channel signal $y_{j}$ in parallel MRI is given by the Fourier transform of magnetization image $\rho$ weighted by the sensitivities of the receive coils $c_{j}$ :

$$
y_{j}(\vec{k})=\int_{\Omega} \mathrm{d} \vec{r} \rho(\vec{r}) c_{j}(\vec{r}) e^{-i 2 \pi \vec{k} \vec{r}}
$$

Here, $\vec{k}$ is the sampling trajectory in the Fourier domain ( $\mathrm{k}$ space), and $\Omega$ is a compactly supported region inside $\mathcal{R}^{2}$ for two-dimensional or $\mathcal{R}^{3}$ for three-dimensional imaging experiments. The extend of $\Omega$ (field-of-view) in the image domain defines the bandwidth of the signal in k-space. If $\mathrm{k}$ space is sampled according to the corresponding Nyquist rate it is referred to as fully sampled. As the signal equation is valid only for short durations, the acquisition of all required k-space samples has to be split into a repeated series of individual measurements, which makes the measurement process very time consuming. The main objective in parallel MRI is to reconstruct diagnostic images while reducing the number of samples as much as possible. Fig. 11 shows various common sampling schemes and Fig. 2 shows individual coil images and sensitivities for a modern 32-channel receiver-coil array.
The signals for all channels are highly correlated. In fact, it can be shown that the space of ideal signals $y_{j}(\vec{k})$ is a subspace of all band-limited k-space signals. Parallel imaging exploits this to reduce the number of samples below the Nyquist limit. The subspace of ideal signals can be characterized as a Reproducing Kernel Hilbert Space with a matrix-valued reproducing kernel given by:

$$
K_{s t}(\vec{u}, \vec{v})=\int_{\Omega} \mathrm{d} \vec{r} c_{s}(\vec{r}) \overline{c_{t}(\vec{r})} e^{-i 2 \pi(\vec{u}-\vec{v}) \vec{r}} .
$$

For known sensitivities $c_{j}$, the reproducing kernel can be computed and standard approximation theory then yields a complete sampling theory with optimal formulas for interpolation and error bounds [1].

More commonly, image reconstruction is formulated as a linear inverse problem and a regularized solution is defined as the minimizer of the following optimization problem [2], [3]:

$$
\underset{x}{\operatorname{argmin}} \sum_{j}\left\|P \mathcal{F} S_{j} x-y_{j}\right\|_{2}^{2}+R(x)
$$

Here, $S_{j}$ is the multiplication with the sensitivity $c_{j}, \mathcal{F}$ the Fourier transform, and $P$ the sampling operator, and $R(x)$ a regularization term. Most state-of-the art algorithms for MRI are based on numerical optimization of the discretized functional and often also combine parallel imaging with concepts from compressed sensing to further reduce the number of samples [4], [5].

\section{Autocalibrating Parallel Imaging}

In practice, the coil sensitivities $c_{j}$ are not known. Many techniques have been developed for autocalibration of the sensitivities from a fully-sampled (calbration) region $y_{\text {calib }}$ in the center of k-space parallel imaging. Early schemes estimated the coil sensitivities directly from low-resolution images obtained as the inverse Fourier transform of the calibration region, but these methods are not optimal and need 


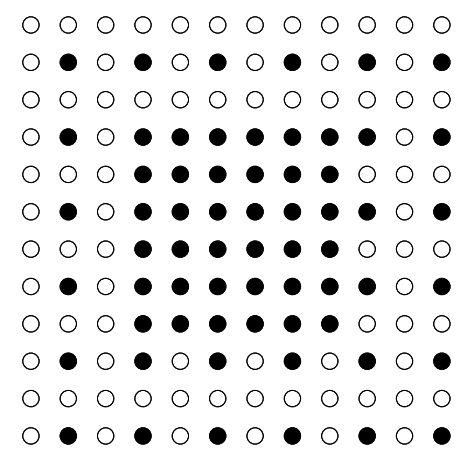

Cartesian sampling with calibration region

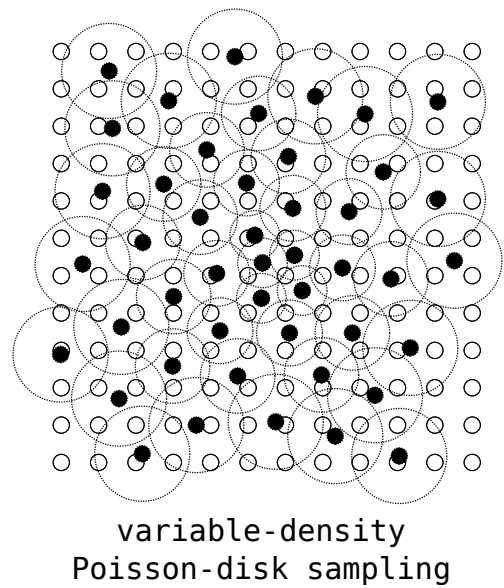

Fig. 1. Typical sampling schemes used in parallel MRI (from left to right): Cartesian sampling with acceleration (undersampling) and a fully-sampled calibration region in the k-space center, variable-density Poisson-disk sampling, and radial sampling. Black dots represent acquired samples.

a high number of additional samples. In the following, we will discuss ESPIRiT as a recent subspace-based approach for autocalibration [6].

Calibration can be formulated using the calibration matrix $\mathcal{C}\left\{y_{\text {calib }}\right\}$, which is constructed as a block-Hankel matrix from fully sampled calibration data $y_{\text {calib }}$ by taking overlapping patches of the multi-channel k-space as rows. Because patches are locally correlated in $\mathrm{k}$-space between receive channels, this matrix has a low rank and singular-value decomposition can be used to compute the basis for the signal subspace $V_{\|}$and for the noise subspace $V_{\perp}$ of patches in k-space.

Given a calibration matrix, highly accurate sensitivities can be recovered using the ESPIRiT algorithm: The null-space condition $V_{\perp} V_{\perp}^{H} R_{r} \hat{y}=0$ for patches $R_{r} \hat{y}$ of the unknown kspace $\hat{y}$ ( $r$ enumerating all patches) yields an overdetermined system of equations which can be solved in the least-squares sense using the normal equations:

$$
\sum_{r} R_{r}^{H} V_{\perp} V_{\perp}^{H} R_{r} \hat{y}=0
$$

This can be rewritten as a matrix-valued convolution $\mathcal{W}$ that reproduces an ideal fully-sampled k-space ( $M$ is the number of samples in a patch):

$$
\underbrace{M^{-1} \sum_{r} R_{r}^{H} V_{\|} V_{\|}^{H} R_{r}}_{\mathcal{W}} y=y
$$

Combining this equation $\mathcal{W} y=y$ with the ideal signal model $y=\mathcal{F} S \rho$ and applying an inverse Fourier transform yields $\mathcal{F}^{-1} \mathcal{W} \mathcal{F} S=1 S$ where $\rho \neq 0$. In other words, the coil sensitivities appear as eigenvectors to the eigenvalue one of the operator $\mathcal{F}^{-1} \mathcal{W} \mathcal{F}$. As this operator is pointwise in the image domain, it can be efficiently be computed. Interestingly, in the case of certain inconsistencies in the data the algorithm produces multiple sets of eigenvector maps to the eigenvalue one. This can be exploited for more robust parallel MRI by using an extended optimization problem for image reconstruction:

$$
\underset{x^{i}}{\operatorname{argmin}} \sum_{j}\left\|P \mathcal{F} \sum_{i} S_{j}^{i} x^{i}-y_{j}\right\|_{2}^{2}+\sum_{i} R\left(x^{i}\right)
$$

Here, $S_{j}^{i}$ correspond to multiple sets $c_{j}^{i}$ of sensitivity maps, and $x^{i}$ are multiple sets of images which then have to be combined in a post-processing step.

\section{Calibrationless Parallel Imaging}

For random or non-Cartesian sampling and when a fully sampled calibration region is not available, calibrationless methods are required. For example, exploiting the low rankness of the calibration matrix $\mathcal{C}\{y\}$ missing k-space samples can be recovered using structured low-rank matrix completion 【7]:

$$
\underset{\hat{y}}{\operatorname{argmin}}\|y-P \hat{y}\| \quad \text { with } \quad \operatorname{rank} \mathcal{C}\{\hat{y}\} \leq L
$$

A solution of this problem can be approximated using Cadzow's algorithm [8], although this is computationally expensive because it requires a large SVD in each iteration. Reconstruction can also be understood directly as a bilinear inverse problem related to multi-channel blind deconvolution [9]-[11]. The regularized non-linear inversion (NLINV) algorithm [11] jointly estimates the image $x$ and the coil sensitivities $c_{j}$ based on the formulation as a non-linear inverse problem:

$$
\begin{aligned}
\underset{x, c_{j}}{\operatorname{argmin}} \sum_{j} & \left\|y_{j}-P \mathcal{F}\left\{c_{j} x\right\}\right\|_{2}^{2}+ \\
& \frac{\alpha}{2}\left(\sum_{j}\left\|W c_{j}\right\|_{2}^{2}+\|x\|_{2}^{2}\right)
\end{aligned}
$$

Here, $W$ a weighting matrix that penalizes high Fourier coefficients to exploit the fact that sensitivities are smooth functions. Fig. 3 shows an experimental example. Inspired by 


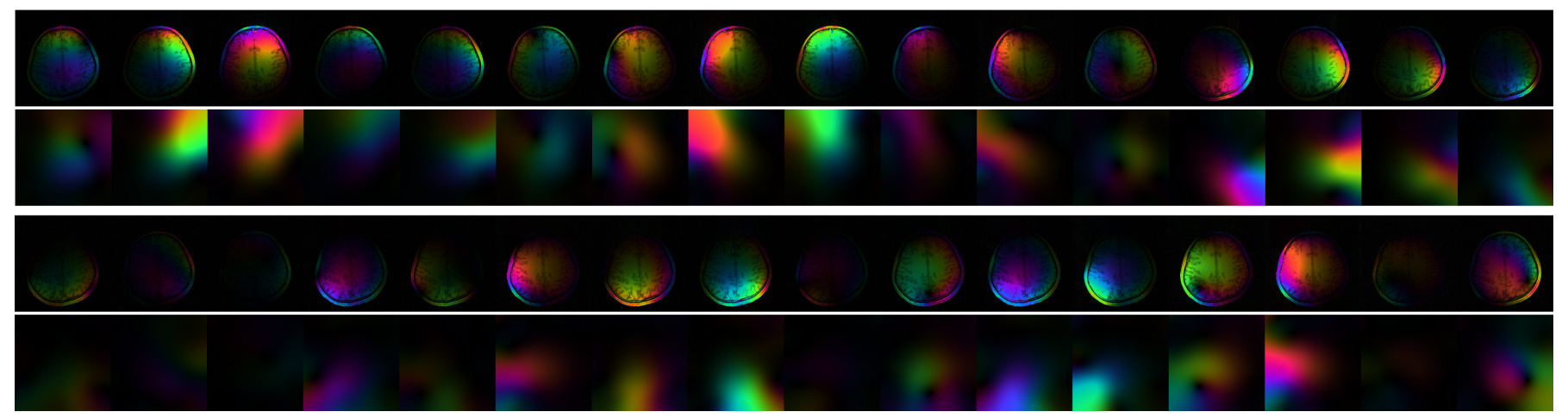

Fig. 2. MRI of a human brain. All 32 channels of a modern receiver-coil array have been reconstructed separately from fully sampled Fourier data (top rows). The corresponding sensitivity map of each coil has been estimated with the NLINV algorithm (bottom rows). The color encodes the argument of the complex-valued maps.

ESPIRiT, this algorithm can be extended to multiple images and sets of sensitivities [12]:

$$
\begin{aligned}
\underset{x, c_{j}}{\operatorname{argmin}} \sum_{j} & \left\|y_{j}-P \mathcal{F}\left\{\sum_{i} c_{j}^{i} x^{i}\right\}\right\|_{2}^{2}+ \\
& \frac{\alpha}{2}\left(\sum_{i, j}\left\|W c_{j}^{i}\right\|_{2}^{2}+\sum_{i}\left\|x^{i}\right\|_{2}^{2}\right)
\end{aligned}
$$

By introducing new transformed variables $u$ and $v$, and a linear operator $\mathcal{A}$ which maps the outer product $X=u v^{T}$ to $P \mathcal{F}\left\{c_{j} x\right\}$, it can be shown that this relaxed version of the algorithm is related to a (convex) structured low-rank matrix recovery problem with nuclear-norm regularization [13].
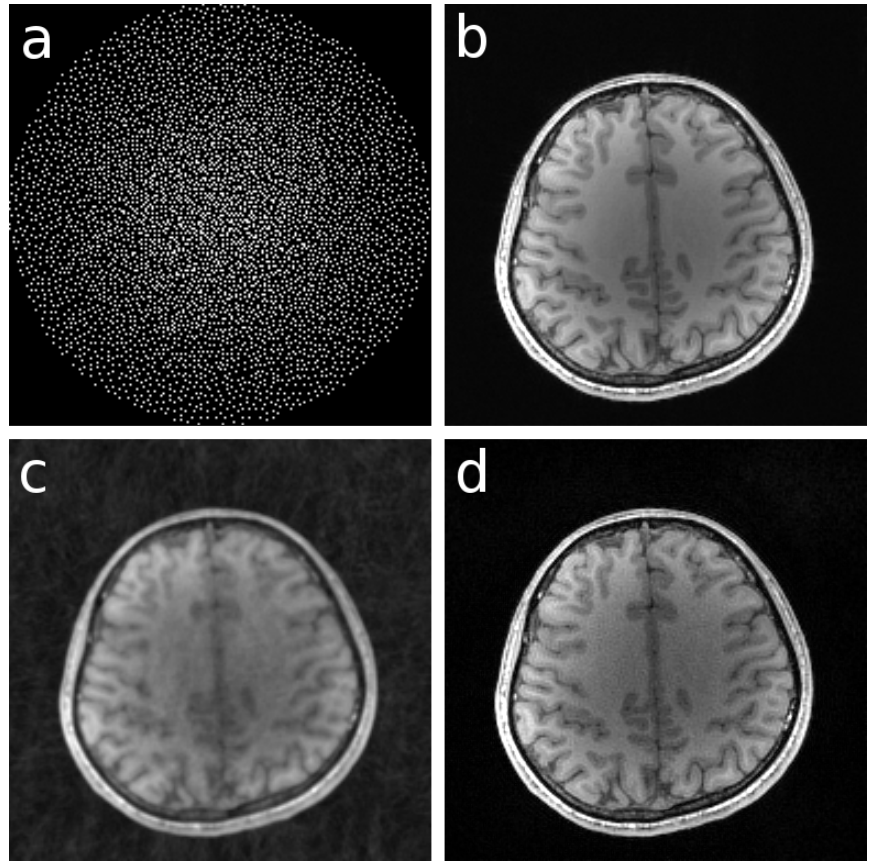

Fig. 3. Calibrationless parallel imaging using regularized non-linear inversion: a: Variable-density Poisson-disc sampling pattern with six-fold undersampling. b: Reference image reconstructed from fully-sampled data c: Image reconstructed from zero-filled data d: Image reconstructed using regularized non-linear inversion

\section{CONCLUSION}

While parallel MRI with known sensitivities is a linear inverse problem for which results from sampling theory and efficient algorithms are readily available, autocalibrating parallel imaging is related to multi-variate multi-channel blind deconvolution and still an active area of research.

\section{REFERENCES}

[1] V. Athalye, M. Lustig, and M. Uecker, "Parallel magnetic resonance imaging as approximation in a reproducing kernel hilbert space," Inverse Problems, vol. 31, p. 045008, 2015.

[2] K. P. Pruessmann, M. Weiger, P. Börnert, and P. Boesiger, "Advances in sensitivity encoding with arbitrary k-space trajectories," Magn Reson Med, vol. 46, pp. 638-651, 2001.

[3] M. Uecker, "Parallel magnetic resonance imaging," in MRI: Physics, Image Reconstruction, and Analysis, A. Majumdar and R. Ward, Eds. CRC Press, 2015, pp. 73-92.

[4] M. Lustig, D. L. Donoho, and J. M. Pauly, "Sparse MRI: The application of compressed sensing for rapid MR imaging," Magn Reson Med, vol. 58, pp. 1182-1195, 2007.

[5] K. T. Block, M. Uecker, and J. Frahm, "Undersampled radial MRI with multiple coils. Iterative image reconstruction using a total variation constraint," Magn Reson Med, vol. 57, pp. 1086-1098, 2007.

[6] M. Uecker, P. Lai, M. J. Murphy, P. Virtue, M. Elad, J. M. Pauly, S. S Vasanawala, and M. Lustig, "ESPIRiT - an eigenvalue approach to autocalibrating parallel MRI: Where SENSE meets GRAPPA," Magn Reson Med, vol. 71, pp. 990-1001, 2014.

[7] P. Shin, P. Larson, M. Ohliger, M. Elad, J. Pauly, D. Vigneron, and M. L. $\mathrm{M}$, "Calibrationless parallel imaging reconstruction based on structured low-rank matrix completion." Magn Reson Med, vol. 72, pp. 959-970, 2014.

[8] J. Cadzow, "Signal enhancement: a composite property mapping algorithm." IEEE Trans Acoust, Speech, Signal Process, vol. 36, pp. 49-61, 1988.

[9] L. Ying and J. Sheng, "Joint image reconstruction and sensitivity estimation in SENSE (JSENSE)," Magn Reson Med, vol. 57, pp. 11961202, 2007.

[10] F. Bauer and S. Kannengiesser, "An alternative approach to the image reconstruction for parallel data acquisition in MRI," Math Methods Appl Sci, vol. 30, pp. 1437-1451, 2007.

[11] M. Uecker, T. Hohage, K. T. Block, and J. Frahm, "Image reconstruction by regularized nonlinear inversion - Joint estimation of coil sensitivities and image content," Magn Reson Med, vol. 60, pp. 674-682, 2008.

[12] H. Holme, F. Ong, S. Rosenzweig, R. Wilke, M. Lustig, and M. Uecker, "ENLIVE: A Non-Linear Calibrationless Method for Parallel Imaging using a Low-Rank Constraint," in Proc Intl Soc Mag Reson Med, vol. 25, Honolulu, 2017, p. 5160.

[13] B. Recht, M. Fazel, and P. A. Parrilo, "Guaranteed Minimum-Rank Solutions of Linear Matrix Equations via Nuclear Norm Minimization," SIAM Review, vol. 52, pp. 471-501, 2010. 